\title{
The Coordinated Development of Industry, Population and Space in Beijing-Tianjin-Hebei Urban Agglomeration
}

\author{
Liu Jie, Jiang Feng*, Li Zhihui \\ Department of International Business, Beijing Union University, Beijing, 100025, China \\ "Corresponding author.Email: ruqinhu@126.com
}

\begin{abstract}
Since the Beijing-Tianjin-Hebei integration strategy has been upgraded to the national strategy, the integration of Beijing-Tianjin-Hebei has made significant progress in several key areas, especially in the industry, ecology and transportation. The core support of integration development has been established. However, there are still gaps in the coordinated development between the population and industry, the population and space. By constructing the industry- population- space development index system, and using the coupling coordination degree function, the coupling coordination degree of industry, population and space of Beijing-Tianjin-Hebei Urban Agglomeration is calculated from 2000 to 2018. The results show that the comprehensive development level of industry-populationspace in Beijing-Tianjin-Hebei Urban Agglomeration is on the rise as a whole. The development process includes three stages: population oriented, industry oriented and coordinated development of industry, population and space. The rapid development of the industrial system is the main reason for the improvement of the coupling coordination degree of the three systems, while the slow growth of the population level restricts the further improvement of the coupling coordination degree of the three systems. Therefore, it is necessary to guide the rationalization of the spatial distribution of industrial functions in urban agglomerations, further improve the level of social security and public services, and promote the coordinated development of urban population, industry and space.
\end{abstract}

Keywords: Coordinated development, Beijing-Tianjin-Hebei, population, industry, space

\section{INTRODUCTION}

Since the strategy of Beijing-Tianjin-Hebei integrated development has been elevated to national strategy, Beijing-Tianjin-Hebei Region integrated development has gain significant progress in multiple major fields, especially in the industry, ecology and transportation ${ }^{[1]}$. The core support of integration development has been established. From the strategic aspect, with issuing and implementation of programming, the top-level design and the strategic plan is becoming continuously more clear and the strategic layout has basically completed. From the concrete aspect, in the past five year, the integration effect of Beijing-Tianjin-Hebei Region has been strengthened continuously, traffic integration has been promoted continuously, the cooperation in ecological environment protection has been deepen continuously, the quality of regional environment has been improved apparently and industrial upgrading and transfer has been boosted continuously. Though Beijing-Tianjin-Hebei region integrated development has gained remarkable effect, there are still many problems, especially there are serious problems in the aspect of coordinated development between population and industry, population and space. In order to solve the uncoordinated development of Beijing-Tianjin-Hebei urban agglomeration, the coordinated development of the industry, population and space should be measured and analyzed.

\section{THEROETICAL FOUNDATION}

As for the development of industry, population and space of urban agglomeration, existing studies mainly study from three aspects from the perspective of research. One is to analyze the impact of single factor on regional economic development from the perspective of industry, population or spatial system, such as the imbalance of industrial structure ${ }^{[2]}$, the decline of demographic dividend ${ }^{[3]}$ and the imbalance of spatial 
land use ${ }^{[4]}$. The second is to analyze the impact of the changes in the relationship between industry, population and spatial factors on local economic development, such as the impact of industrial transformation and population aging ${ }^{[5]}$, the mismatch between population urbanization and spatial urbanization ${ }^{[6]}$, and the mismatch between industrial transfer and spatial resources $^{[7]}$. The third is to comprehensively analyze the impact of the comprehensive development and change of industry, population and space system on local economy, such as the research on the coupling and coordination of industry, population and space in urban agglomeration of Yangtze River Delta, Pearl River Delta and Chengdu Chongqing urban agglomeration.

In terms of research methods, there are mainly four methods. Firstly, the econometric model is used to analyze the urban development ${ }^{[8]}$. Secondly, the coupling coordination model is used to analyze the urban development ${ }^{[9]}$. Thirdly, the urban development analysis and evaluation index system is constructed ${ }^{[10]}$. Fourthly, the urban development is studied by using the geographical spatial analysis method ${ }^{[11]}$.

With the deepening of the research in related fields, people gradually realize that industry, population and space are important components of regional economic development. The lag of any system will lead to system imbalance, and ultimately affect the sustainable and healthy development of urban agglomeration. However, at present, the research on the urban agglomeration of Beijing-Tianjin-Hebei focuses on the problems of population, resources and ecological environment, while there are few researches on the coordinated development of industry, population and space. Therefore, with the coupling coordination degree model, this paper analyzes the overall development level and coupling coordination degree of industry, population and space of Beijing-Tianjin-Hebei Urban Agglomeration by building index system and entropy method, and using relevant urban data of Beijing-

Table 1 Index system for coordinated development of urban industry-population-space

\begin{tabular}{|c|c|c|}
\hline system & index & nature \\
\hline \multirow{3}{*}{ industry } & Per capita GDP (ten thousand yuan) & + \\
& Number of industrial enterprises & + \\
& Proportion of primary industry in GDP (\%) & + \\
& The proportion of secondary industry in GDP (\%) & + \\
& The proportion of tertiary industry in GDP (\%) & + \\
\hline
\end{tabular}

Tianjin-Hebei Urban Agglomeration from 2000 to 2018, so as to provide reference for the further optimization and implementation of Beijing-Tianjin-Hebei integrated development strategy.

\section{DETERMINATION OF THE INDEX SYSTEM}

Based on the existing research and fully considering the actual development of the Beijing-Tianjin-Hebei urban agglomeration, an industry-population-spatial evaluation index system has been established. In terms of industrial indicators, the per capita GDP is selected to indicate the level of urban economic development based on existing literature. At the same time, the proportion of the primary, secondary, and tertiary industries in the regional GDP and the proportion of the actual use of foreign capital in the regional GDP are selected to represent the industrial structure and the degree of external dependence. Finally, the number of industrial enterprises above designated size is selected to represent the main body of micro enterprises. In terms of population indicators, the number of permanent residents and population density in the city at the end of the year are selected to reflect the size of the urban population and the regional population distribution, the proportion of employees in the secondary and tertiary industries and the urban registered unemployment rate are selected to represent the urban employment status, and the urbanization rate is selected to represent the level of urbanization. In terms of spatial development, we select urban land area and built-up area to represent urban spatial development and construction, select urban green area to represent urban environmental protection and greening, finally, the per capita urban road area and the number of buses owned by 10,000 people are selected to reflect the urban transportation infrastructure construction status. 


\begin{tabular}{|c|c|c|}
\hline \multirow{3}{*}{ population } & $\begin{array}{c}\text { Number of permanent residents at the end of the year }(10,000 \text { people) } \\
\left.\text { Population density (person/ } \mathrm{km}^{2}\right)\end{array}$ & + \\
& $\begin{array}{c}\text { The proportion of employees in the secondary and tertiary industries }(\%) \\
\text { Urban registered unemployment rate } \\
\text { Urbanization rate }(\%)\end{array}$ & + \\
\hline space & Urban land area $\left(\mathrm{km}^{2}\right)$ & + \\
& Urban built-up area $\left(\mathrm{km}^{2}\right)$ & + \\
& Urban green area $\left(\mathrm{km}^{2}\right)$ & + \\
& Urban road area per capita $\left(\mathrm{m}^{2}\right)$ & + \\
\hline
\end{tabular}

\section{EVALUATION METHOD OF COUPLING COORDINATION DEGREE}

\subsection{Coupling Degree Model}

$$
C_{t}=\left\{\frac{f\left(U_{1 t}\right) \times f\left(U_{2 t}\right) \times f\left(U_{3 t}\right)}{\Pi\left[f\left(U_{i t}\right)+f\left(U_{j t}\right)\right]}\right\}^{\frac{1}{3}}
$$

Where $\mathrm{i}, \mathrm{j}=1,2,3 ; \mathrm{i} \neq \mathrm{j}$. $C_{t}$ represents the degree of coupling, and the value is between $0-1$. The larger the value, the tighter the coupling effect, indicating a high degree of mutual influence between industry, population and space.

\subsection{Coupling Coordination Degree Model}

The coupling degree model can only describe the degree of mutual influence between systems, but it is impossible to determine whether the system is high-level coupling or low-level coupling. Therefore, the coupling coordination degree model is further used to accurately measure the coupling coordination status between systems. The coupling coordination function is:

$$
R_{t}=\left(C_{t} \times T_{t}\right)^{\frac{1}{3}}
$$

In the formula, $R_{t}$ represents the degree of coupling coordination, and $T_{t}$ represents the comprehensive evaluation index, namely:

$$
T_{t}=\alpha f\left(U_{1 t}\right)+\beta f\left(U_{2 t}\right)+\gamma f\left(U_{3 t}\right)
$$

If the $R_{t}$ value is high, it means that the systems are at a high level of coupling and coordination, interacting and influencing each other at a high level. Because industry, population and space are equally important to the development of urban agglomerations, set $\alpha=\beta=\gamma=1 / 3$. Based on the previous research, the stages of coordinated and coordinated development of different cities are set according to the degree of coupling and coordination. If the coupling coordination degree is below 0.75 (excluding 0.75), it is a low-level coupling coordinated development stage; $0.75-0.8$ is a medium level coupling and coordinated development stage (excluding 0.8); and 0.8 and above is a high-level coupling and coordinated development stage.

\section{EMPIRICAL ANALYSIS}

It can be seen from Figure 1 that the comprehensive coupling coordination degree among the industrial, population and spatial systems of Beijing-Tianjin-Hebei has basically realized the transformation from the stage of medium and low-level coupling and coordinated development to the stage of medium and high-level coupling and coordinated development. The coupling coordination degree can be roughly divided into three stages. The first stage was from 2000 to 2007. The coupling coordination degree was between 0.742 and 0.746 , but lower than 0.742 in 2002 , showing a trend of decreasing first and then rising. The second stage was from 2008 to 2013. The coupling coordination degree showed a steep decline and then a steady increase trend, with the highest reaching 0.7457 . The third stage was from 2014 to 2018 , the coupling coordination degree increased significantly, which shows that the BeijingTianjin-Hebei integration strategy has promoted the coordinated and orderly development of BeijingTianjin-Hebei Urban Agglomeration.

The change trend of coupling coordination degree among industry, population and space is roughly the same as that of comprehensive coupling coordination degree of the three. According to figure 2, the coupling coordination degree of industry and population in Beijing-Tianjin-Hebei region increased steadily from 2000 to 2007. The coupling coordination degree of industry and space showed a rising trend in fluctuation, and the coupling coordination degree of population and space showed a downward trend in fluctuation. In terms of specific scores, the degree of coupling coordination between industry and space system is the lowest, but the gap between the coupling degree of the industry and population and the population and space system is gradually narrowing. The lag of spatial development is the main reason for restricting the coupling coordination 
degree of the three major systems in this period. From 2008 to 2013, the coupling coordination degree of population and space has increased steadily, and the coupling coordination degree of industry and population has fluctuated obviously. The coupling coordination degree of industry and space has increased significantly, exceeding that of population and space, and even temporarily exceeding the coupling coordination of industry and population in 2013. From 2014 to 2018, the coupling coordination degree between industry, population and space has been continuously improved. The increase of coupling coordination degree between industry and population, industry and space is larger than that of population and space, which indicates that the rapid development of industrial system is the main reason for the improvement of coupling coordination degree of three systems, while the slow growth of population level restricts the further improvement of that of three systems.

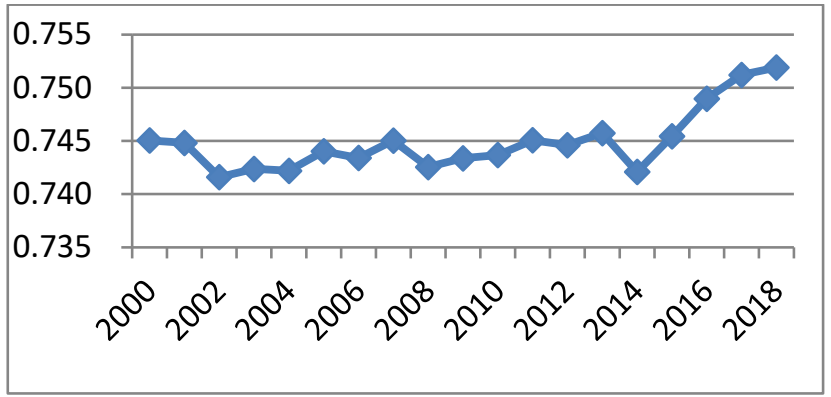

Figure.1 coupling coordination degree of the industrial, population and space in Beijing-Tianjin-Hebei region

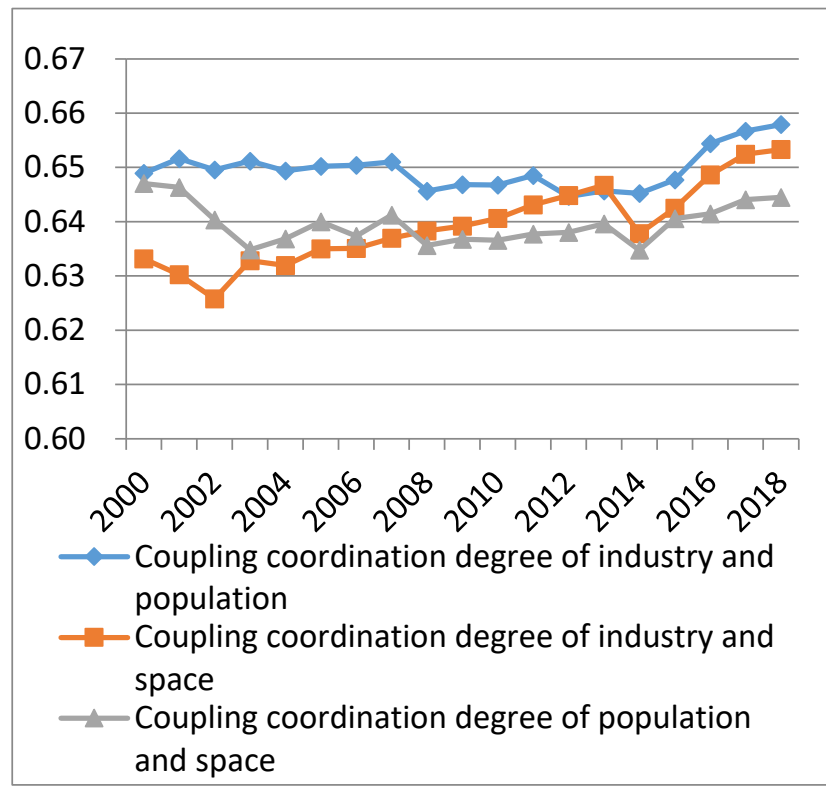

Figure. 2 coupling coordination degree of between the industry and population, industry and space, and population and space in Beijing-Tianjin-Hebei region

\section{CONCLUSIONS}

\subsection{Main Conclusions}

From the perspective of time sequence, the development of the industry-population-space of Beijing-Tianjin-Hebei urban agglomeration shows an upward trend. The evolution process includes three stages. The first stage is the urban development mode with population development as the main driving force during the period of 2000-2007. However, due to the influence of China's accession to WTO on the population development level of Beijing-Tianjin-Hebei Urban Agglomeration, the coordinated development level of cities shows a downward trend and then an upward trend. The second stage is from 2008 to 2013. Under the background of the government's overall economic adjustment, a development mode with industrial development as the main driving force has been formed. However, affected by the U.S. subprime mortgage crisis, the number of unemployed has increased, and the level of population development has decreased in fluctuations. The third stage is from 2014 to 2018. As the Beijing-Tianjin-Hebei integration strategy officially becomes the national level strategy, the coupling coordination degree is constantly improved. However, the coupling coordination degree of population and space is still low, which restricts the coupling coordinated development of urban agglomeration.

\subsection{Recommendations}

Firstly, promote the coupling coordination of urban population and industry and their positive influence on each other, so as to improve the ability of Hebei cities to attract talents. It is necessary to strengthen the top-level design, and promote the establishment of the communication mechanism among the three governments, so as to connect the transferred industries of Beijing and Tianjin with Hebei effectively. Adjust the registered residence management of different administrative divisions, and connect the public service systems with each other, so that a large number of floating population living in city will get the corresponding public services such as education, health and social security, so as to ensure the fair treatment of migrant workers, promote the improvement of human capital of migrant workers, and improve the comprehensive measures of industry and population.

Secondly, adhering to the concept of sustainable development, promote the coupling coordinated development of urban industry and space. According to the development stage of each city in the BeijingTianjin-Hebei Urban Agglomeration, combined with the resource factor endowment of each city, further improve the coordination and cooperation mechanism, fiscal and 
tax coordination mechanism and market integration mechanism of the coordinated development of BeijingTianjin-Hebei Urban Agglomeration, and thus form a hierarchical industrial chain and effective industrial linkage mechanism of theBeijing-Tianjin-Hebei Urban Agglomeration, and accelerate the industrial transformation and upgrading, to realize the complementary and coordinated development of the industrial development within the Beijing-Tianjin-Hebei Urban Agglomeration.

Finally, adhere to the development for the interests of the people, promote the coupling coordinated development of urban population and space. In the period of further development Beijing-Tianjin-Hebei integration strategy, promote the coordinated development of population and space in urban agglomeration, increase the supply of public goods and services, and realize the coordinated development of population and space, economy and resources and environment of urban agglomeration.

\section{ACKNOWLEDGMENT}

This research was financially supported by Beijing Young Top Talents Training Program (CIT\&TCD201704068).

\section{REFERENCES}

[1] Liu Jie, Jiang Feng, Qian Chunli. Systematic research on coordinated development of Beijing Tianjin Hebei [J]. China Soft Science, 2020 (04): 142-153.

[2] Wang Baijie, Guo Xin. Local government behavior, "resource curse" and industrial structure imbalance: evidence from survey data of 43 resource-based prefecture level cities [J]. Journal of Shanxi University of Finance and Economics, 2017, 39 (06): 64-75.

[3]Cai Fang. Future demographic dividend: the development of the source of China's economic growth [J]. China Population Science, 2009 (01): $2-10+111$

[4]Wang Siyuan, liujiyuan, zhang zengxiang, zhou quanbin, zhaoxiaoli. Analysis of the spatial and temporal characteristics of land use in China [J]. Journal of Geography, 2001 (06): 631-639.

[5] Wang Wei, Liu Yufei, Peng Dongdong. Research on the upgrading effect of industrial structure of population aging $[\mathrm{J}]$. China Industrial Economy, 2015 (11): 47-61.

[6] Zhao Guoqing, Wu Xueping. Spatial dynamic mechanism and effect of urbanization in China: Based on the data of 2869 county units in the sixth census [J]. China Soft Science, 2017 (02): 76-87.
[7] Xie Chengyang, Zhou Haibo, Hu Hanhui. Spatial mismatch of factor resources and economic efficiency loss in industrial transfer: a study based on the survey data of Jiangsu traditional enterprises [J]. China Industrial Economy, 2014 (12): 130-142.

[8] Xiao Zhouyan. Study on spatial effect of consistency of population and economic distribution in China [J]. Population research, 2013, 37 (05): 42-52.

[9] Li Qiuying, Fang Chuanglin, Wang Shaojian, Wang Yang. Coordinated development and spatial pattern of population urbanization and spatial urbanization in Shandong Province [J]. Regional Research and Development, 2015,34 (01): 31-36.

[10] Zeng Rong, Wei Yiming, Fan Ying, Li Zhijie. Analysis and evaluation index system of coordinated development of population, resources, environment and economy in Beijing [J]. China Management Science, 2000 (S1): 310-317.

[11] Sun Pingjun, Ding Sibao. Spatial differentiation of urbanization in Northeast China from the perspective of population economy space [J]. Economic Geography, 2011,31 (07): 1094-1100. 